\title{
OPEN Epidemiologic trends in cancer-related emergency department utilization in Korea from 2015 to 2019
}

\author{
Sun Young Lee $\rrbracket^{1,2}$, Young Sun Ro $\mathbb{1}^{2,3,4}$, Sang Do Shin $\mathbb{1}^{2,3}$ \& Sungwoo Moon $\rrbracket^{4,5}$
}

It is inevitable for cancer patients to visit the emergency department (ED) for symptoms of cancer itself and various treatment-related complications. As the prevalence of cancer increases along with cancer survival rates, the number of ED visits of cancer patients may increase. This study aimed to investigate the epidemiologic trends and characteristics of cancer-related ED visits. A cross-sectional study was conducted for all ED visits nationwide between 2015 and 2019. The characteristics of cancer- and non-cancer-related ED visits were compared, and the cancer type and primary reason for ED visits were investigated for cancer-related ED visits. The age- and sex-standardized incidence rate per 100,000 population was calculated. Among 44,983,523 ED visits for 5 years, 1,372,119 (3.1\%) were cancer-related. Among cancer-related ED visits, $54.8 \%$ led to hospitalization including $5.1 \%$ in ICU, and $9.5 \%$ died in the hospital. The age- and sex-standardized incidence rates of cancer-related ED visits per 100,000 population increased from 521.8 in 2015 to 642.2 in 2019 ( $p$-for-trends, <0.01), and rates of cancer-related hospital admission via ED were 309.0 in 2015 and 336.6 in 2019 (p-for-trends, 0.75). The most common cancer types were lung cancer $(14.7 \%)$, liver cancer $(13.1 \%)$, and colorectal cancer $(11.5 \%)$. The most common primary reasons of cancer-related ED visits were pneumonia (3.6\%), gastroenteritis (2.7\%), fever (2.6\%), abdominal pain (2.4\%), and ileus (2.1\%). Cancer-related ED visits accounted for $3.1 \%$ of all ED visits, with 1.37 million cases over five years. The incidence rate of cancer-related ED visits has increased year by year, with high hospitalization and mortality rates, and the burden of cancer-related ED visits will continue to increase as the prevalence increases.

Cancer is an important public health burden globally ${ }^{1}$. Around the world, the 5 -year prevalence of cancer reaches 50 million people, and 19 million new cases occur annually ${ }^{2}$. Cancer patients frequently visit hospitals for complex cancer treatments such as chemotherapy and radiation therapy, as well as various problems that occur during care ${ }^{3}$. In case of sudden worsening of symptoms or side effects of cancer treatment after the end of inpatient treatment, it is inevitable for cancer patients to visit the emergency departments (EDs) ${ }^{4}$. The ED is an important medical resource for patients with acute medical illnesses that requires a lot of manpower and equipment to operate 5 .

The number of patients visiting the ED is increasing rapidly, especially in countries with an aging population and high prevalence of chronic diseases ${ }^{6,7}$. EDs should be prepared to provide adequate care in response to emergency diseases where timely treatment is critical for prognosis, such as acute myocardial infarction and severe injury ${ }^{5,8}$. At the same time, non-urgent treatment for chronic diseases or ambulatory care-sensitive conditions is also provided in the ED, due to the nature of the ED operating $24 \mathrm{~h}$ a day, 7 days a week ${ }^{5,9}$. As such, it is necessary to monitor the characteristics of patients visiting the ED in order to ensure that the ED can function properly for patients with emergency conditions.

Cancer patients are bound to visit the ED for symptoms caused by the disease itself and side effects from cancer treatment ${ }^{4,10}$. Cancer-related ED visits accounted for 3.7 to $4.2 \%$ of all ED visits ${ }^{10,11}$. Approximately $27 \%$ of patients with solid tumors who received chemotherapy had at least one visit to $\mathrm{ED}^{4}$. Cancer patients stayed

${ }^{1}$ Public Healthcare Center, Seoul National University Hospital, Seoul, Korea. ${ }^{2}$ Laboratory of Emergency Medical Services, Seoul National University Hospital Biomedical Research Institute, Seoul, Korea. ${ }^{3}$ Department of Emergency Medicine, Seoul National University Hospital, Seoul, Korea. ${ }^{4}$ National Emergency Medical Center, National Medical Center, Seoul, Korea. ${ }^{5}$ Department of Emergency Medicine, Korea University Ansan Hospital, Gyeonggi, Korea. ${ }^{\square}$ email: Ro.youngsun@gmail.com 
in the ED longer, received more radiological tests, were more likely to present acute care conditions in the ED, and were hospitalized more after treatment, compared to non-cancer patients ${ }^{10-12}$. Cancer is not a traditional emergency disease to be treated in the ED as it is a chronic disease ${ }^{13}$. Nevertheless, cancer patients have no choice but to visit the ED sometimes, and use a lot of ED resources when they do. However, not much is known about characteristics of cancer-related ED visits.

As the prevalence of cancer increases along with cancer survival rates, the number of ED visits of cancer patients may increase. However, it is not known what proportion of cancer patients visit the ED nor why. Research on cancer-related ED visits is important both for purposes to improve the quality of cancer treatment by understanding the clinical needs of cancer patient, and for reducing unnecessary ED visits by investigating the epidemiologic characteristics of cancer-related ED visits ${ }^{10}$. The aim of this study is to investigate the epidemiologic trends and characteristics of cancer-related ED visits using a nationwide ED database. In detail, we aimed to investigate the scale and disposition of ED visits according to the cancer type and primary cause of ED visits, and explore factors associated with intensive care unit (ICU) admission of cancer-related ED visits.

\section{Methods}

Study design and data sources. A cross-sectional study was conducted with the National Emergency Department Information System (NEDIS) database. NEDIS is a nationwide ED based database constructed by the Ministry of Health and Welfare in 2013 and operated by the National Emergency Medical Center. NEDIS collected clinical and administrative information of patients visiting EDs from a total 402 EDs across the country in $2019^{14}$. All patient-related information including demographics, prehospital, ED, and disposition information was automatically transferred from each ED to a central government server within 14 days of the discharge date from the ED or hospital. The trained coordinators designated in each institution managed the data uploading process.

Study setting. Korea has a population of approximately 50 million people in 17 provinces including eight metropolitan cities. National Health Insurance (NHI) was implemented in Korea in 1989. NHI is a single insurer operated by Korean government covering the entire population. Since NHI covered all inpatient, outpatient, and emergency care, Korea has high accessibility to medical care including emergency care ${ }^{15,16}$. In addition, due to NHI's policy of expanding benefit coverage for serious diseases, cancer patients have a lower rate of out-ofpocket expenditure and have higher access to medical care ${ }^{17}$. Under this healthcare system, EDs are open to all beneficiaries without restriction ${ }^{15}$. EDs are classified into 3 levels according to resource and capacity, which are defined by the Ministry of Health and Welfare. In 2020, 38 regional EDs (Level 1), 125 local EDs (Level 2), and 239 emergency facilities (Level 3) were operated.

The National Cancer Center manages the Korea Central Cancer Registry (KCCR) in accordance with the Cancer Control Act, and reports an "Annual report of cancer incidence, survival and prevalence in Korea" since $2007^{18}$. As the population ages, the incidence and prevalence of cancer in Korea are on the rise. In 2017, there were 0.23 million newly diagnosed cancer cases, and 5-year cancer prevalence was 1.87 million ${ }^{18}$.

Study population. The study population was patients who visited EDs from January 2015 to December 2019. Cases with unknown age were excluded. The NEDIS data had multiple diagnosis codes (up to 40) at the time of discharge from the ED or hospital using the International Classification of Diseases, 10th edition (ICD10). The term cancer-related ED visit was defined as if any of the diagnosis codes entered in NEDIS database were ICD-10 code C00-C97 (malignant neoplasms).

Outcome measures and variables. The study outcomes were ICU admission, hospital admission via ED, and in-hospital mortality.

We obtained the following information from the NEDIS database: patients' demographics (age, sex, and insurance types (NHI, Medical Aid, and others)), prehospital and ED information (region of ED (metropolitan or not), route of ED visit (direct, transfer-in, and others), use of EMS when visiting ED, ED visit times, level of ED (level 1, 2 and 3)), ED disposition (ED discharge diagnosis code and disposition status (discharge, transfer-out, ward admission, ICU admission, death, and else), and hospital disposition in case of hospitalization (hospital discharge diagnosis code and disposition status (discharge, transfer-out, death, and else)).

Cancer type was classified based on the ICD-10 code ${ }^{18}$. If multiple cancer codes were recorded in one visit, it was classified as the cancer type of the code listed in the previous order. The primary reason of ED visits of cancer patients was defined as non-cancer diagnosis codes among diagnosis codes of NEDIS database. Cases with only cancer diagnosis were excluded from the analysis of the primary reason of ED visits. If multiple noncancer diagnoses were entered, the first listed diagnosis code was used for the analysis.

Statistical analysis. Descriptive analysis was conducted to compare the characteristics of cancer-related and non-cancer-related visits in ED. Categorical variables were shown by counts and proportion and tested by Chi-square test. Continuous variables were shown by medians and quartiles and tested by Wilcoxon rank-sum test.

To investigate the annual trends of cancer-related ED visits, the crude and the age- and sex-standardized incidence rates of cancer-related ED visits and study outcomes per 100,000 population were calculated using a direct standardization method using the 2020 mid-year census population as a standard population ${ }^{19}$. Yearly trends of age- and sex-standardized incidence rates were tested by p-for-trend test for a total of cancer-related ED visits and top 5 cancer-types. 
Among cancer-related ED visits, a multivariable logistic regression analysis was conducted to investigate associated factors with ICU admission. Adjusted odds ratios (AORs) and confidence intervals (CIs) were calculated. Data management and statistical analyses were conducted using SAS software version 9.4 (SAS Institute Inc., Cary, NC, USA) and Stata version 13.1 (StataCorp, College Station, TX). Statistical significance was taken as $\mathrm{P}<0.05$.

Ethical statement. This study was approved by the Institutional Review Boards of Seoul National University Hospital (approval No. SNUH-2012-104-1183), and the requirement for informed consent was waived due to the retrospective nature of this study. All methods were performed in accordance with relevant guidelines and regulations.

Patient and public involvement statement. The National Emergency Medical Center under the Ministry of Health and Welfare was involved in the design and conduct of this research, but it was not possible to involve patients in our research.

\section{Results}

Characteristics of the study population. During the 5 years of study period, the total number of ED visits was 44,984,017. Excluding 494 cases of unknown age, 44,983,523 cases were included in the study.

Among them, there were 1,372,119 cancer-related ED visits, which accounted for $3.1 \%$ of all ED visits. The median age of cancer-related visits was 65 years old, older than that of non-cancer-related visits (41 years old). Among cancer-related ED visits, 58.0\% visited metropolitan ED and 18.1\% used EMS. A total of 54.8\% of cancer-related visits were hospitalized, and 5.1\% were admitted to the ICU, which were higher than those of non-cancer-related ED visits (hospital admission $16.8 \%$ and ICU admission $2.3 \%$ ). The in-hospital mortality of cancer-related ED visits was $9.5 \%$, which was higher than $1.0 \%$ of non-cancer-related ED visits (Table 1).

Trends and epidemiologic characteristics of cancer-related ED visits. The crude incidence rate of cancer-related ED visits per 100,000 populations increased from 444.9 in 2015 to 620.8 in 2019 (p<0.01). The age- and sex-standardized incidence rate of cancer-related ED visits per 100,000 populations also increased from 521.8 in 2015 to 642.2 in 2019 ( $\mathrm{p}<0.01$ ). The annual age- and sex-standardized rates of hospital outcomes for cancer-related ED visits were presented in Fig. 1 (in 2015 and 2019, 309.0 and 336.6 for cancer-related hospital admission via ED, 28.3 and 32.0 for cancer-related ICU admission via ED, and 55.8 and 57.5 for in-hospital mortality). Analysis by cancer type was presented as supplement (Supplement Fig. 1).

For cancer-related ED visits, the most common cancer type was lung cancer, accounting for $14.7 \%$ of all cancer-related ED visits. Among them, 54.0\% were hospitalized and $14.6 \%$ died in hospitals. The second most common ED visiting cancer type was liver cancer (13.1\%), and followed by colorectal cancer (11.5\%), stomach cancer (9.0\%), and pancreas cancer (6.0\%). Cancer types with high hospitalization rates were gallbladder and biliary tract cancer (59.4\%), leukemia (54.9\%), and non-Hodgkin lymphoma (54.1\%) (Table 2).

As for the primary reason for visiting the ED, 865,793 cases (63.1\%) with a non-cancer diagnosis code were analyzed, excluding 506,326 cases (36.9\%) that were diagnosed only with cancer code during cancer-related ED visits. The most common reason for ED visit was pneumonia, accounting for 3.6\% of all cancer-related ED visits, of which $73.4 \%$ were hospitalized and $23.9 \%$ died in the hospital. The next most common primary reasons were gastroenteritis (2.7\%), fever (2.6\%), abdominal pain (2.4\%), and ileus and intestinal obstruction (2.1\%) (Table 3).

Factors associated with ICU admission among cancer-related ED visits. Of the cancer-related ED visits, 69,948 cases (5.1\%) were admitted to the ICU after ED treatment. Significantly increasing the odds for ICU admission were the over 65 years old compared to the 19-64 years old (AOR, 95\% CI; 1.40, 1.38-1.42), the Medical Aid as health insurance compared to the NHI (AOR, 95\% CI; 1.17, 1.14-1.20), transfer-in visit compared to the direct visit (AOR, 95\% CI; 3.90, 3.83-3.98), EMS use (AOR, 95\% CI; 3.88, 3.81-3.95), and night time (AOR, 95\% CI; 1.16, 1.14-1.18). Compared with other diagnoses, pneumonia (AOR, 95\% CI; 1.81, 1.77-1.87) and dyspnea (AOR, 95\% CI; 1.35, 1.27-1.43) showed significant increases of odds for ICU admission (Table 4).

\section{Discussion}

Using nationwide ED database, we investigated the trends and epidemiologic characteristics of cancer-related ED visits. In Korea, there were 1,372,119 cancer-related ED visits nationwide during the 5 years from 2015 to 2019 , accounting for $3.1 \%$ of all ED visits. More than half of cancer-related visits were hospitalized, and 9.5\% died in the hospital. Incidence rate of cancer-related ED visits increased during the study period (age- and sex-standardized incidence rate per 100,000 populations, 521.8 in 2015 and 642.2 in 2019, p <0.01). The most common cancer types in cancer-related ED visits were lung cancer, liver cancer, and colorectal cancer. The most common primary reasons of ED visits were pneumonia, gastroenteritis, and fever. As the incidence and prevalence of cancer increase, cancer-related ED visits are also on the rise, and the increase in cancer-related ED visits with such high rates of hospitalization and mortality shows that the burden of the ED is increasing.

Cancer-related ED visits constituted 3.1\% of all ED visits in Korea, slightly lower than that of 3.7 to $4.2 \%$ in the US studies ${ }^{10,11}$. More than $50 \%$ of cancer-related visits were hospitalized, similar to the US, which was about three times the hospitalization rate of non-cancer-related visits (54.8\% versus $16.8 \%$ ), and the ICU admission rates were also more than twice that of non-cancer-related visits (5.1\% versus $2.3 \%)$. These results show that the burden on the ED for cancer-related visits is very high. The most common primary reason of cancer-related ED visits was pneumonia, and more than $70 \%$ of patients diagnosed with pneumonia were hospitalized. Diabetes, 


\begin{tabular}{|c|c|c|c|c|c|c|}
\hline & \multicolumn{2}{|l|}{ Total } & \multicolumn{2}{|l|}{ Non-cancer } & \multicolumn{2}{|l|}{ Cancer } \\
\hline & $\mathbf{N}$ & $\%$ & $\mathbf{N}$ & $\%$ & $\mathbf{N}$ & $\%$ \\
\hline Total & $44,983,523$ & 100.0 & $43,611,404$ & 100.0 & $1,372,119$ & 100.0 \\
\hline \multicolumn{7}{|l|}{ Age, year } \\
\hline $0-18$ & $10,200,978$ & 22.7 & $10,172,301$ & 23.3 & 28,677 & 2.1 \\
\hline $19-64$ & $25,672,168$ & 57.1 & $25,035,938$ & 57.4 & 636,230 & 46.4 \\
\hline $65-120$ & $9,110,377$ & 20.3 & $8,403,165$ & 19.3 & 707,212 & 51.5 \\
\hline Median (IQR) & \multicolumn{2}{|l|}{$42(21-60)$} & \multicolumn{2}{|l|}{$41(20-60)$} & \multicolumn{2}{|l|}{$65(55-75)$} \\
\hline Sex, female & $21,790,304$ & 48.4 & $21,224,004$ & 48.7 & 566,300 & 41.3 \\
\hline \multicolumn{7}{|l|}{ Insurance } \\
\hline National Health Insurance & $39,171,555$ & 87.1 & $37,938,191$ & 87.0 & $1,233,364$ & 89.9 \\
\hline Medical Aid & $2,611,816$ & 5.8 & $2,486,013$ & 5.7 & 125,803 & 9.2 \\
\hline Others & $3,200,152$ & 7.1 & $3,187,200$ & 7.3 & 12,952 & 0.9 \\
\hline Metropolitan & $19,781,628$ & 44.0 & $18,986,485$ & 43.5 & 795,143 & 58.0 \\
\hline \multicolumn{7}{|l|}{ Route of ED visit } \\
\hline Direct & $41,141,931$ & 91.5 & $40,107,509$ & 92.0 & $1,034,422$ & 75.4 \\
\hline Transfer-in & $3,186,182$ & 7.1 & $2,955,923$ & 6.8 & 230,259 & 16.8 \\
\hline Others & 655,410 & 1.5 & 547,972 & 1.3 & 107,438 & 7.8 \\
\hline EMS use & $6,998,753$ & 15.6 & $6,750,443$ & 15.5 & 248,310 & 18.1 \\
\hline \multicolumn{7}{|l|}{ ED visit time } \\
\hline Night & $23,241,110$ & 51.7 & $22,799,287$ & 52.3 & 441,823 & 32.2 \\
\hline Weekend & $17,133,056$ & 38.1 & $16,762,081$ & 38.4 & 370,975 & 27.0 \\
\hline \multicolumn{7}{|l|}{ Level of ED } \\
\hline Level 1 & $8,248,885$ & 18.3 & $7,835,584$ & 18.0 & 413,301 & 30.1 \\
\hline Level 2 & $16,922,243$ & 37.6 & $16,695,909$ & 38.3 & 226,334 & 16.5 \\
\hline Level 3 & $19,812,395$ & 44.0 & $19,079,911$ & 43.7 & 732,484 & 53.4 \\
\hline \multicolumn{7}{|l|}{ Disposition of ED } \\
\hline Discharge & $35,616,831$ & 79.2 & $35,057,229$ & 80.4 & 559,602 & 40.8 \\
\hline Transfer-out & 768,668 & 1.7 & 721,068 & 1.7 & 47,600 & 3.5 \\
\hline Admission, total & $8,096,264$ & 18.0 & $7,343,973$ & 16.8 & 752,291 & 54.8 \\
\hline Death & 241,129 & 0.5 & 230,036 & 0.5 & 11,093 & 0.8 \\
\hline Others & 260,631 & 0.6 & 259,098 & 0.6 & 1,533 & 0.1 \\
\hline Admission, ICU & $1,092,039$ & 2.4 & $1,022,091$ & 2.3 & 69,948 & 5.1 \\
\hline In-hospital mortality & 567,858 & 1.3 & 437,467 & 1.0 & 130,391 & 9.5 \\
\hline
\end{tabular}

Table 1. Demographics of cancer-related and non-cancer-related emergency department visits from 2015 to 2019. $E D$ emergency department, $I Q R$ interquartile range, $E M S$ emergency medical service.

agranulocytosis, and ileus and intestinal obstruction were also hospitalized in more than $70 \%$. A combination of cancer and chronic disease such as diabetes can increase the risk of hospitalization after an ED visit ${ }^{20}$. Agranulocytosis is a typical side effect that occurs after chemotherapy, and ileus is common after laparotomy ${ }^{21,22}$. The high hospitalization rate for these reasons means that cancer patients who visit the ED with these symptoms need close monitoring and treatment. Conversely, ascites had a relatively low hospitalization rate (27.8\%) compared to others in the list of top 10 reasons. Some of the malignant ascites had to be managed through outpatient treatment rather than in the $\mathrm{ED}^{23}$.

The National Cancer Center in Korea publish national cancer statistics every year ${ }^{18}$. According to the most recently published 2017 cancer statistics, the 5-year cancer prevalence of Korea was 1.87 million, while top five cancers with the highest prevalence were thyroid, stomach, colorectal, breast, and prostate cancers ${ }^{18}$. Unlike these national prevalence data, the top five cancers that led to ED visits were lung, liver, colorectal, stomach, and pancreas cancer. Thyroid cancer accounted for $21.7 \%$ of the national cancer prevalence, but only $1.0 \%$ in cancer-related ED visits. In contrast, lung cancer accounted for the only $4.5 \%$ of the national cancer prevalence, but $14.7 \%$ in cancer-related ED visits. Cancer patients visit the ED when symptoms suddenly worsen or develop symptoms that cannot be treated on an outpatient basis. Because different cancer types have different symptoms and treatment side effects, there may be differences in the ED visits and hospital outcomes according to the cancer 
A Crude incidence rates

(per 100,00o population)

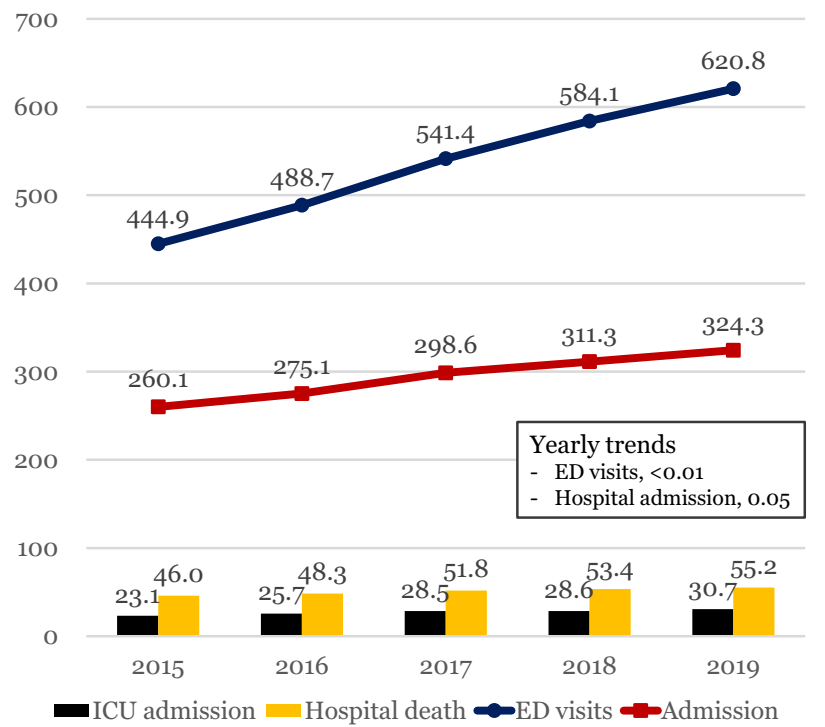

B Age- and sex-standardized incidence rates

(per 100,000 population)

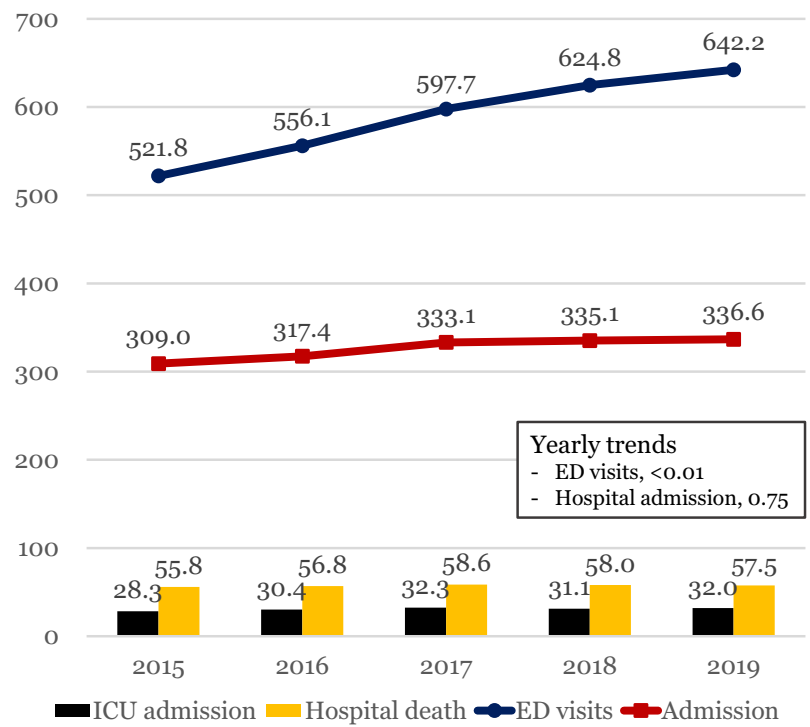

Figure. 1. Trends of the crude and age- and sex-standardized incidence rates of cancer-related ED visits per 100,000 population from 2015 to 2019. (A) Crude incidence rate, (B) age-and sex-standardized incidence rate.

\begin{tabular}{|c|c|c|c|c|c|c|}
\hline \multirow[b]{2}{*}{ Cancer type (ICD-10 code) } & \multicolumn{2}{|l|}{ Total } & \multicolumn{3}{|c|}{ ED disposition (\%) } & \multirow[b]{2}{*}{ In-hospital mortality (\%) } \\
\hline & $\mathbf{N}$ & $\%$ & Ward & ICU & Death & \\
\hline Total & $1,372,119$ & 100 & 49.7 & 5.1 & 0.8 & 9.5 \\
\hline Lung cancer (C33-C34) & 201,367 & 14.7 & 54.0 & 5.6 & 1.3 & 14.6 \\
\hline Liver cancer (C22) & 179,177 & 13.1 & 49.9 & 7.3 & 1.1 & 12.2 \\
\hline Colorectal cancer $(\mathrm{C} 18-20)$ & 157,654 & 11.5 & 48.2 & 5.4 & 0.6 & 7.4 \\
\hline Others & 138,700 & 10.1 & 45.9 & 4.4 & 0.6 & 8.2 \\
\hline Stomach cancer (C16) & 123,272 & 9.0 & 51.6 & 5.5 & 0.8 & 9.2 \\
\hline Pancreas cancer (C25) & 82,077 & 6.0 & 51.2 & 3.3 & 0.9 & 10.8 \\
\hline Breast cancer (C50) & 81,417 & 5.9 & 39.6 & 1.8 & 0.4 & 4.8 \\
\hline Gallbladder and biliary tract cancer (C23-24) & 73,371 & 5.3 & 59.4 & 4.1 & 0.7 & 9.0 \\
\hline Leukemia (C91-95) & 49,899 & 3.6 & 54.9 & 4.4 & 0.6 & 9.7 \\
\hline Non-Hodgkin lymphoma (C82-86, C96) & 42,164 & 3.1 & 54.1 & 4.9 & 0.6 & 8.8 \\
\hline Prostate cancer (C61) & 39,383 & 2.9 & 42.0 & 6.1 & 0.8 & 6.7 \\
\hline Ovarian cancer (C56) & 31,159 & 2.3 & 48.2 & 2.1 & 0.5 & 5.2 \\
\hline Bladder cancer (C67) & 30,907 & 2.3 & 46.7 & 5.0 & 0.5 & 6.4 \\
\hline Brain and CNS cancer (C70-72) & 25,571 & 1.9 & 50.0 & 9.8 & 0.5 & 6.6 \\
\hline Cervix uteri cancer (C53) & 23,002 & 1.7 & 45.4 & 3.2 & 0.6 & 5.5 \\
\hline Multiple myeloma (C90) & 22,321 & 1.6 & 53.4 & 6.4 & 0.7 & 9.1 \\
\hline Esophagus cancer (C15) & 20,697 & 1.5 & 50.6 & 5.1 & 1.1 & 10.5 \\
\hline Lip, oral cavity and pharynx cancer (C00-14) & 18,182 & 1.3 & 41.0 & 4.6 & 1.2 & 8.7 \\
\hline Kidney cancer (C64) & 17,495 & 1.3 & 50.0 & 5.7 & 0.6 & 8.1 \\
\hline Thyroid cancer (C73) & 14,304 & 1.0 & 35.1 & 4.4 & 0.3 & 3.3 \\
\hline
\end{tabular}

Table 2. Cancer type and hospital outcomes among patients with cancer-related emergency department visits. $E D$ emergency department, ICU intensive care unit. 


\begin{tabular}{|c|c|c|c|c|c|c|}
\hline \multirow[b]{2}{*}{ Diagnoses* } & \multicolumn{2}{|l|}{ Total } & \multicolumn{3}{|c|}{ ED disposition (\%) } & \multirow[b]{2}{*}{ In-hospital mortality (\%) } \\
\hline & $\mathbf{N}$ & $\%$ & Ward & ICU & Death & \\
\hline Pneumonia & 48,839 & 3.6 & 73.4 & 12.3 & 0.9 & 23.9 \\
\hline Gastroenteritis & 37,714 & 2.7 & 50.5 & 2.4 & 0.1 & 4.6 \\
\hline Fever & 36,329 & 2.6 & 53.2 & 2.8 & 0.1 & 4.7 \\
\hline Abdominal pain & 33,051 & 2.4 & 49.4 & 2.3 & 0.1 & 5.6 \\
\hline Ileus and intestinal obstruction & 28,622 & 2.1 & 78.7 & 3.7 & 0.1 & 5.1 \\
\hline Agranulocytosis & 26,781 & 2.0 & 79.2 & 2.9 & 0.2 & 4.7 \\
\hline Diabetes & 19,327 & 1.4 & 79.3 & 6.4 & 0.5 & 15.6 \\
\hline Dyspnea & 16,171 & 1.2 & 55.9 & 8.6 & 1.4 & 21.1 \\
\hline Pleural effusion & 14,410 & 1.1 & 63.7 & 4.2 & 0.4 & 14.5 \\
\hline Ascites & 14,097 & 1.0 & 27.8 & 1.1 & 0.1 & 6.9 \\
\hline
\end{tabular}

Table 3. Top 10 reason for emergency department visits and hospital outcomes among patients with cancerrelated visits. ED emergency department, $I C U$ intensive care unit. ${ }^{\star}$ Pneumonia, J12-18; fever, R50; abdominal pain, R10; ileus and intestinal obstruction, K56; gastroenteritis, A90, K52, K29; agranulocytosis, D70; dyspnea, R06; diabetes, E11-14; pleural effusion, K90-91, C78.2; ascites, R18, C78.6.

type. In terms of the management of medical resources, this is the reason why research on healthcare utilization for cancer patients by cancer type is needed in addition to nationwide prevalence statistics.

Traditionally, ED is a department that responds to sudden emergencies or time-sensitive conditions ${ }^{13,24,25}$. Cancer itself causes symptoms such as acute abdominal pain and dyspnea, and cancer treatments also cause side effects such as neutropenia ${ }^{21,26}$. For that reasons, cancer patients have to visit the hospital frequently, including $\mathrm{ED}^{26,27}$. Previously, little was known about the epidemiologic characteristics of cancer-related ED visits, as the main stage of the oncologist in charge of treating cancer patients are outpatient clinic and wards ${ }^{27}$. In response to the increasing trends of cancer-related ED visits, the American Academy of Emergency Medicine advocated in 2016 that four areas of research were needed to improve emergency care for cancer patients; (1) the collection of epidemiologic data, (2) care of patients with febrile neutropenia, (3) acute events such as dyspnea, and (4) palliative care in the ED setting ${ }^{28}$. This study, which presents epidemiologic data of cancer-related ED visits in Korea, is the first step. In addition, the studies of avoidable ED visits are also important, given the increasing number of cancer-related ED visits ${ }^{29}$. Some cancer patients may need hospice care rather than ED treatment ${ }^{30}$, and some cancer patients may not have visited the ED if they have been prescribed appropriate medications to control their symptoms ${ }^{31}$.

As cancer incidence and prevalence increase, number of cancer-related ED visits is also on an increasing trend. This is the first nationwide study on the epidemiologic trends of cancer-related ED visits in Korea, using a large, nationally representative database. Based on this epidemiologic data, further research is needed to reduce the burden of ED of cancer patients and improve cancer management in the ED.

This study has several limitations. Firstly, this study classified cancer-related ED visits based on the diagnosis codes entered into the NEDIS database. If there was a cancer diagnosis code in either one of the ED discharge codes or hospital discharge codes, it was classified as cancer-related ED visit. If the cancer diagnosis code is missing, the cancer-related ED visits may be misclassified. However, since patients with cancer diagnosis code have high coverage benefit in Korea, it is very unlikely that the cancer diagnosis was omitted. Secondly, NEDIS database lacks information on cancer staging or diagnosis/treatment status. It is unknown what cancer stage the patients are on, whether they are receiving active treatment, and when they were diagnosed with cancer. In-depth studies of various ED visits in cancer patients are needed to understand the associations between characteristics of cancer-related ED visits and their prognosis. Thirdly, the primary reasons for the ED visits were classified according to the order of non-cancer diagnosis code. If multiple non-cancer diagnoses were entered, only the first listed diagnosis code was used for the analysis, as the principal diagnosis coded first. However, there is a possibility of misclassification. In addition, since NEDIS database does not contain laboratory data, detailed information about the diagnosis is absent.

In conclusion, there were 1.37 million cancer-related ED visits over five years, which is increasing every year. The most common cancer types were lung cancer, liver cancer, and stomach cancer. The common primary reasons of cancer-related ED visits were pneumonia, gastroenteritis, and fever. More than half of cancer-related ED visits were hospitalized and in-hospital mortality rate was at $9.5 \%$. Research and strategies are needed to reduce the growing burden of cancer-related ED visits and improve the ED care of cancer patients. 


\begin{tabular}{|c|c|c|c|c|c|c|}
\hline \multirow{3}{*}{$\begin{array}{l}\text { Characteristics } \\
\text { Total }\end{array}$} & \multirow{3}{*}{$\begin{array}{l}\text { Total } \\
\mathbf{N} \\
1,372,119\end{array}$} & \multicolumn{2}{|c|}{$\begin{array}{l}\text { ICU } \\
\text { admission }\end{array}$} & \multicolumn{3}{|c|}{ Odds ratio } \\
\hline & & \multirow{2}{*}{\begin{tabular}{|l|}
$\mathrm{N}$ \\
69,948
\end{tabular}} & \multirow{2}{*}{$\begin{array}{l}\% \\
5.1\end{array}$} & \multirow[t]{2}{*}{ Ratio } & \multicolumn{2}{|c|}{$95 \% \mathrm{CI}$} \\
\hline & & & & & & \\
\hline \multicolumn{7}{|l|}{ Age } \\
\hline $0-18$ & 28,677 & 555 & 1.9 & 0.66 & 0.61 & 0.72 \\
\hline $19-64$ & 636,230 & 23,427 & 3.7 & 1.00 & & \\
\hline $65-120$ & 707,212 & 45,966 & 6.5 & 1.40 & 1.38 & 1.42 \\
\hline \multicolumn{7}{|l|}{ Sex } \\
\hline Male & 805,819 & 46,151 & 5.7 & 1.00 & & \\
\hline Female & 566,300 & 23,797 & 4.2 & 0.81 & 0.80 & 0.83 \\
\hline \multicolumn{7}{|l|}{ Insurance } \\
\hline National Health Insurance & $1,233,364$ & 60,958 & 4.9 & 1.00 & & \\
\hline Medical Aid & 125,803 & 7,939 & 6.3 & 1.17 & 1.14 & 1.20 \\
\hline Others & 12,952 & 1,051 & 8.1 & 1.54 & 1.44 & 1.65 \\
\hline \multicolumn{7}{|l|}{ Metropolitan } \\
\hline No & 576,976 & 33,550 & 5.8 & 1.22 & 1.20 & 1.24 \\
\hline Yes & 795,143 & 36,398 & 4.6 & 1.00 & & \\
\hline \multicolumn{7}{|l|}{ Route of ED visit } \\
\hline Direct & $1,034,422$ & 43,635 & 4.2 & 1.00 & & \\
\hline Transfer-in & 230,259 & 23,790 & 10.3 & 3.90 & 3.83 & 3.98 \\
\hline Others & 107,438 & 2,523 & 2.3 & 0.95 & 0.91 & \begin{tabular}{|l|l}
0.99 \\
\end{tabular} \\
\hline \multicolumn{7}{|l|}{ EMS use } \\
\hline No & $1,123,809$ & 43,580 & 3.9 & 1.00 & & \\
\hline Yes & 248,310 & 26,368 & 10.6 & 3.88 & 3.81 & 3.95 \\
\hline \multicolumn{7}{|l|}{ Time of ED visits } \\
\hline Day time & 930,296 & 45,417 & \begin{tabular}{|l|}
4.9 \\
\end{tabular} & 1.00 & & \\
\hline Night time & 441,823 & 24,531 & 5.6 & 1.16 & 1.14 & 1.18 \\
\hline \multicolumn{7}{|l|}{ Week of ED visits } \\
\hline Weekday & $1,001,144$ & 52,630 & 5.3 & 1.00 & & \\
\hline Weekend & 370,975 & 17,318 & 4.7 & 0.97 & 0.95 & \begin{tabular}{|l|}
0.99 \\
\end{tabular} \\
\hline \multicolumn{7}{|l|}{ Level of ED } \\
\hline Level 1 & 413,301 & 24,298 & 5.9 & 1.00 & & \\
\hline Level 2 & 732,484 & 35,614 & 4.9 & 0.90 & 0.88 & 0.91 \\
\hline Level 3 & 226,334 & 10,036 & 4.4 & 0.83 & 0.81 & \begin{tabular}{l|l} 
& 0.85 \\
\end{tabular} \\
\hline \multicolumn{7}{|l|}{ Primary diagnoses } \\
\hline Pneumonia & 48,839 & 6000 & 12.3 & 1.82 & 1.77 & 1.87 \\
\hline Fever & 36,329 & 1009 & 2.8 & 0.56 & 0.53 & 0.60 \\
\hline Abdominal pain & 33,051 & 745 & 2.3 & 0.44 & 0.41 & 0.47 \\
\hline Ileus and intestinal obstruction & 28,622 & 1062 & 3.7 & 0.64 & 0.60 & 0.68 \\
\hline Gastroenteritis & 37,714 & 915 & 2.4 & 0.50 & 0.47 & 0.54 \\
\hline Agranulocytosis & 26,781 & 775 & 2.9 & 0.73 & 0.68 & 0.79 \\
\hline Dyspnea & 16,171 & 1388 & 8.6 & 1.35 & 1.27 & 1.43 \\
\hline Diabetes & 19,327 & 1234 & 6.4 & 1.02 & 0.96 & 1.08 \\
\hline Pleural effusion & 14,410 & 604 & 4.2 & 0.68 & 0.63 & 0.74 \\
\hline Ascites & 14,097 & 149 & 1.1 & 0.25 & 0.22 & 0.30 \\
\hline Others & $1,096,778$ & 56,067 & 5.1 & 1.00 & & \\
\hline
\end{tabular}

Table 4. Multivariable logistic regression model for intensive care unit admission of cancer-related emergency department visits. $E D$ emergency department, IQR interquartile range, $E M S$ emergency medical service.

\section{Data availability}

The data of this study were obtained from the National Emergency Medical Center under the Ministry of Health and Welfare in Korea but restrictions apply to the availability of these data and so are not publicly available.

Received: 28 June 2021; Accepted: 1 November 2021

Published online: 09 November 2021 


\section{References}

1. Bray, F., Ferlay, J., Soerjomataram, I., Siegel, R.L., Torre, L.A., \& Jemal, A. Global cancer statistics 2018: GLOBOCAN estimates of incidence and mortality worldwide for 36 cancers in 185 countries. CA Cancer J. Clin. 68(6), 394-424 (2018).

2. Sung, H. et al. Global cancer statistics 2020: GLOBOCAN estimates of incidence and mortality worldwide for 36 cancers in 185 countries. CA Cancer J. Clin. 71(3), 209-249 (2021).

3. Pearce, A. et al. Incidence and severity of self-reported chemotherapy side effects in routine care: A prospective cohort study. PLoS ONE 12(10), e0184360 (2017).

4. Panattoni, L. et al. Characterizing potentially preventable cancer-and chronic disease-related emergency department use in the year after treatment initiation: A regional study. J. Oncol. Pract. 14(3), e176-e185 (2018).

5. Smulowitz, P. B., Honigman, L. \& Landon, B. E. A novel approach to identifying targets for cost reduction in the emergency department. Ann. Emerg. Med. 61(3), 293-300 (2013).

6. Tang, N., Stein, J., Hsia, R. Y., Maselli, J. H. \& Gonzales, R. Trends and characteristics of US emergency department visits, 19972007. JAMA 304(6), 664-670 (2010).

7. Roberts, D. C., McKay, M. P. \& Shaffer, A. Increasing rates of emergency department visits for elderly patients in the United States, 1993 to 2003. Ann. Emerg. Med. 51(6), 769-774 (2008).

8. Ibanez, B. et al. 2017 ESC Guidelines for the management of acute myocardial infarction in patients presenting with ST-segment elevation: The Task Force for the management of acute myocardial infarction in patients presenting with ST-segment elevation of the European Society of Cardiology (ESC). Eur. Heart J. 39(2), 119-177 (2018).

9. Hsieh, V.C.-R., Hsieh, M.-L., Chiang, J.-H., Chien, A. \& Hsieh, M.-S. Emergency department visits and disease burden attributable to ambulatory care sensitive conditions in elderly adults. Sci. Rep. 9(1), 1-9 (2019).

10. Rivera, D. R. et al. Trends in adult cancer-related emergency department utilization: an analysis of data from the nationwide emergency department sample. JAMA Oncol. 3(10), e172450-e (2017).

11. Hsu, J. et al. National characteristics of Emergency Department visits by patients with cancer in the United States. Am. J. Emerg. Med. 36(11), 2038-2043 (2018).

12. Mayer, D. K., Travers, D., Wyss, A., Leak, A. \& Waller, A. Why do patients with cancer visit emergency departments? Results of a 2008 population study in North Carolina. J. Clin. Oncol. 29(19), 2683 (2011).

13. Wallace, D. J. et al. Referral regions for time-sensitive acute care conditions in the United States. Ann. Emerg. Med. 72(2), 147-155 (2018).

14. Pak, Y. S. et al. Effects of emergency care-related health policies during the COVID-19 pandemic in Korea: A quasi-experimental study. J. Korean Med. Sci. 36(16), e121 (2021).

15. Lee, S. Y., Khang, Y.-H. \& Lim, H.-K. Impact of the 2015 middle east respiratory syndrome outbreak on emergency care utilization and mortality in South Korea. Yonsei Med. J. 60(8), 796-803 (2019).

16. Kim, T. H. et al. Association of health insurance with post-resuscitation care and neurological outcomes after return of spontaneous circulation in out-of-hospital cardiac arrest patients in Korea. Resuscitation 135, 176-182 (2019).

17. Kim, S. \& Kwon, S. Impact of the policy of expanding benefit coverage for cancer patients on catastrophic health expenditure across different income groups in South Korea. Soc. Sci. Med. 138, 241-247 (2015).

18. Hong, S. et al. Cancer statistics in Korea: Incidence, mortality, survival, and prevalence in 2017. Cancer Res. Treat. 52(2), 335 (2020).

19. Korea S. Population of the Middle of the Year. https://kosiskr/statHtml/statHtmldo?orgId=101\&tblId=DT_1B040M5. Accessed 26 Dec 2021. (2019).

20. Srokowski, T. P., Fang, S., Hortobagyi, G. N. \& Giordano, S. H. Impact of diabetes mellitus on complications and outcomes of adjuvant chemotherapy in older patients with breast cancer. J. Clin. Oncol. 27(13), 2170 (2009).

21. Crawford, J., Dale, D. C. \& Lyman, G. H. Chemotherapy-induced neutropenia: Risks, consequences, and new directions for its management. Cancer 100(2), 228-237 (2004).

22. Millan, M. et al. Risk factors for prolonged postoperative ileus after colorectal cancer surgery. World J. Surg. 36(1), 179-185 (2012).

23. Becker, G., Galandi, D. \& Blum, H. E. Malignant ascites: Systematic review and guideline for treatment. Eur. J. Cancer 42(5), 589-597 (2006).

24. Thatcher, J. L., Gilseth, T. A. \& Adlis, S. Improved efficiency in acute myocardial infarction care through commitment to emergency department initiated primary PCI. J. Invasive Cardiol. 15(12), 693-698 (2003).

25. Boulanger, J. et al. Canadian stroke best practice recommendations for acute stroke management: Prehospital, emergency department, and acute inpatient stroke care, update 2018. Int. J. Stroke 13(9), 949-984 (2018).

26. Walsh, D. \& Rybicki, L. Symptom clustering in advanced cancer. Support. Care Cancer 14(8), 831-836 (2006).

27. Kirkland, S. W., Garrido-Clua, M., Junqueira, D. R., Campbell, S. \& Rowe, B. H. Preventing emergency department visits among patients with cancer: A scoping review. Support. Care Cancer 28, 4077-4094 (2020).

28. Brown, J. et al. The emergency care of patients with cancer: Setting the research agenda. Ann. Emerg. Med. 68(6), 706-711 (2016).

29. Oh, T. K., Jo, Y. H. \& Choi, J. W. Associated factors and costs of avoidable visits to the emergency department among cancer patients: 1-year experience in a tertiary care hospital in South Korea. Support. Care Cancer 26(11), 3671-3679 (2018).

30. Obermeyer, Z., Clarke, A. C., Makar, M., Schuur, J. D. \& Cutler, D. M. Emergency care use and the Medicare hospice benefit for individuals with cancer with a poor prognosis. J. Am. Geriatr. Soc. 64(2), 323-329 (2016).

31. Adam, R., Clausen, M. G., Hall, S. \& Murchie, P. Utilising out-of-hours primary care for assistance with cancer pain: A semistructured interview study of patient and caregiver experiences. Br. J. Gen. Pract. 65(640), e754-e760 (2015).

\section{Author contributions}

Conceptualization: Dr. S.Y.L. and Dr. Y.S.R. Data curation: Dr. S.Y.L. and Dr. Y.S.R. Formal analysis: Dr. S.Y.L. Funding acquisition: None. Investigation: Dr. S.M. Methodology: Dr. Y.S.R. and Dr. S.M. Software: Dr. S.Y.L. Supervision: Dr. Y.S.R., Dr. S.D.S., and Dr. S.M. Validation: Dr. Y.S.R., Dr. S.D.S., and Dr. S.M. Visualization: Dr. S.Y.L. Writing —original draft: Dr. S.Y.L. Writing—review \& editing: Dr. Y.S.R., Dr. S.D.S., and Dr. S.M. Approval of final manuscript: all authors.

\section{Funding}

No funding was received for conducting this study.

\section{Competing interests}

The authors declare no competing interests. 


\section{Additional information}

Supplementary Information The online version contains supplementary material available at https://doi.org/ 10.1038/s41598-021-01571-1.

Correspondence and requests for materials should be addressed to Y.S.R.

Reprints and permissions information is available at www.nature.com/reprints.

Publisher's note Springer Nature remains neutral with regard to jurisdictional claims in published maps and institutional affiliations.

(c) (i) Open Access This article is licensed under a Creative Commons Attribution 4.0 International License, which permits use, sharing, adaptation, distribution and reproduction in any medium or format, as long as you give appropriate credit to the original author(s) and the source, provide a link to the Creative Commons licence, and indicate if changes were made. The images or other third party material in this article are included in the article's Creative Commons licence, unless indicated otherwise in a credit line to the material. If material is not included in the article's Creative Commons licence and your intended use is not permitted by statutory regulation or exceeds the permitted use, you will need to obtain permission directly from the copyright holder. To view a copy of this licence, visit http://creativecommons.org/licenses/by/4.0/.

(C) The Author(s) 2021 